\title{
A SY-Stematic approach towards understanding stem cell biology
}

\section{Gregor-Alexander Pilz ${ }^{1, *}$ and Marlen Knobloch ${ }^{2, *}$}

\begin{abstract}
The 2nd SY-Stem Symposium - a symposium for 'the next generation of stem cell researchers' - was held on the 21-23 March 2019 at the Vienna BioCenter in Austria. After the great success of the initial SY-Stem meeting in 2018, this year's event again focused on the work of young scientists. Here, we summarize the impressive amount of new research covering stem cell-related fields that was discussed at the meeting.
\end{abstract}

KEY WORDS: Organoids, Pluripotency, Reprogramming, Single cell analysis, Stem cells

\section{Introduction}

Beautiful Vienna hosted the 2nd SY-Stem Symposium, which brought together 245 researchers from 26 different countries. The organizers of the previous SY-Stem meeting, Elly Tanaka, Juergen Knoblich and Ulrich Elling (Institute of Molecular Biotechnology, Austria) were joined this year by IMBA group leaders Kikue Tachibana, BonKyoung Koo and Noelia Urban. Twenty-eight primarily junior researchers, who had just started their own labs, recently transitioned to a permanent position or were senior postdoctoral fellows, presented their work on various stem cell research topics. During two poster sessions, 59 posters were presented. Two keynote lectures, given by Janet Rossant (The Hospital for Sick Children, University of Toronto, Canada) and Jonas Frisen (Karolinska Institute, Sweden), framed the two major topics of the meeting (Fig. 1): how stem cells make 'decisions' during early development and how stem cells function in the adult.

The meeting was organized without any parallel sessions and covered a broad range of topics and model systems. These included embryonic stem cells (ESCs), induced pluripotent stem cells (iPSCs), neural stem cells (NSCs), hematopoietic stem cells (HSCs) as well as organoids; the model systems ranged from mouse, human and marmosets to planarians and plants, which stimulated lively discussions across fields. Below, we summarize the main findings presented at the symposium.

\section{Totipotency, pluripotency and cell identity}

The first two sessions of the meeting revolved around fundamental concepts in development: how do cells transition from a totipotent or pluripotent stage to differentiation; what drives and controls this process and how plastic is it; and how is cell identity established and maintained?

Katsuhiko Hayashi (Kyushu University, Japan) illustrated how oogenesis can be recapitulated in vitro starting from ESCs or iPSCs, using a protocol that has been recently published (Hayashi et al.,

\footnotetext{
${ }^{1}$ Brain Research Institute, University of Zurich, Zurich 8057, Switzerland.

2Department of Physiology, University of Lausanne, Lausanne 1005, Switzerland.

*Authors for correspondence (pilz@hifo.uzh.ch; marlen.knobloch@unil.ch)

iD G.-A.P., 0000-0002-6161-4976; M.K., 0000-0002-7490-0285
}

Received 15 April 2019; Accepted 21 May 2019
2017; Hikabe et al., 2016). In females, oocytes are produced early on and last for the entire reproductive life, and a fundamental unsolved question concerns how these oocytes are kept dormant. Hayashi presented evidence to suggest that dormancy is regulated by the extracellular matrix (ECM), as artificially removing the ECM from ovaries leads to oocyte activation. Furthermore, Hayashi showed that culturing ovaries in a compression chamber keeps them dormant, suggesting that the ECM regulates dormancy through tension. He also presented a fascinating but yet unclear phenomenon: under pressure (from the ECM or the pressure chamber), the nuclei of the oocytes constantly spin. The functional consequences of such nuclear spinning in oocytes will be an interesting topic for future studies.

Germline cells contain a number of epigenetic marks that need to be reset in in order to unleash their developmental potential. How this is achieved and the consequences of differences in epigenetic marks in sperm and oocytes were addressed in two talks covering different systems. Frederic Berger (Gregor Mendel Institute of Molecular Plant Biology, Austria) presented data on how the histone methylation mark H3K27me3 is selectively erased from Arabidopsis sperm chromatin through a mechanism that includes deposition of a sperm-specific histone H3 variant. Deborah Bourc'his (Institute Curie, France) showed that DNA methylation of the oocyte is vital, and that upon ablation of this methylation pattern in oocytes, mutant embryos die at mid-gestation, even when fertilized with wild-type sperm, suggesting crucial imprinting effects mediated by the oocyte. Furthermore, using scRNA-seq in mutant and wild-type oocytes, she showed that the master regulator for zygotic genome activation, Dux, is upregulated in mutants, leading to an upregulation of Dux target genes.

Besides epigenetic marks, transcription factors (TFs) play a key role in early developmental processes. However, during mitosis, TFs are generally evicted from their targets and therefore need to reestablish functional interactions in daughter cells. Two speakers discussed the mechanisms involved in this event. Pablo Navarro (Institut Pasteur, France) showed that, in ESCs, the TF Esrrb, but not other classical pluripotency TFs such as Nanog, Oct4 or Sox2, remains mitotically bound at selected regulatory elements - a process known as mitotic bookmarking. At Esrrb-bookmarked sites, nucleosomes remain ordered, possibly fostering the reassembly of regulatory complexes after mitosis. In contrast, at sites that exhibit loss of TF binding, nucleosomes are profoundly rearranged (Festuccia et al., 2019). Mario Soares, a PhD student in the Castro laboratory (Instituto Gulbenkian de Ciência, Portugal) also described how the TF Brn2, but not Ascl1, remains associated with condensed chromosomes in NSCs. However, in this context, $\mathrm{Brn} 2$ is not engaged in sequence-specific interactions at regulatory elements. Overall, these findings suggest that the global retention of TFs on mitotic chromatin may increase their local concentration to facilitate the search of their targets after mitosis and foster transcriptional reactivation.

TFs also act by binding to specific enhancers. Joshua Brickman (DanStem, University of Copenhagen, Denmark) showed how this mechanism maintains the plasticity of mouse ESCs during their 


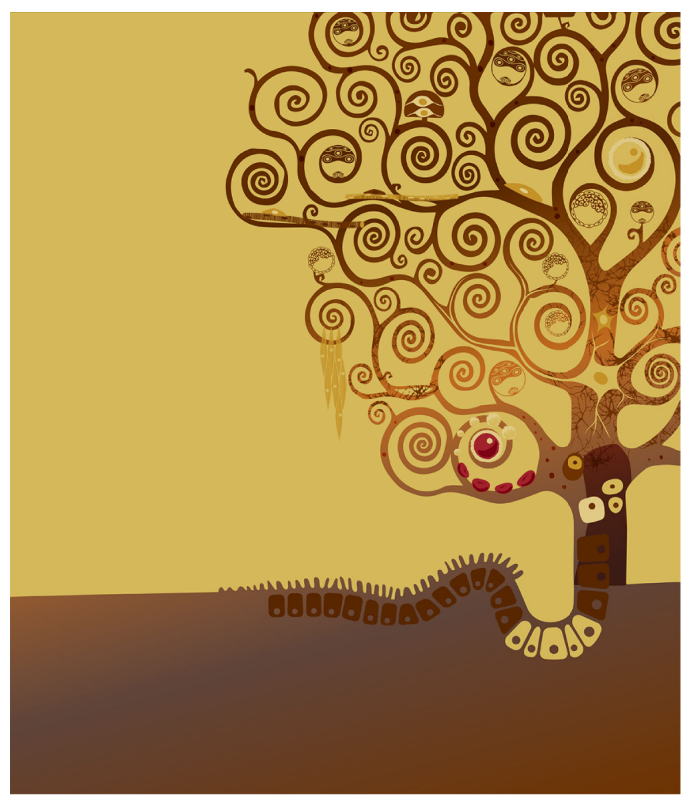

Fig. 1. The tree of life. For the meeting poster, the graphic designer Iza Kaminski (Vienna) illustrated the two main meeting topics - how stem cells make 'decisions' during early embryo development and how stem cells function in the adult using a famous picture by the Austrian painter Gustav Klimt (1862-1918) called 'The Tree of Life'. In her newly designed interpretation of this painting, the spirally rolled tree branches give rise to blastocysts, a structure that had just been observed by Klimt's contemporary biologists, and various differentiated cells.

transition to primitive endoderm. He reported that, despite the transcriptional repression of certain pluripotency genes during differentiation, the TFs that regulate these genes remain stable at the protein level and can remain bound to their corresponding enhancers, thus rapidly reactivating the pluripotency state if required. Less well-known players during early development are the so-called transposable elements (Bourque et al., 2018): pieces of DNA that are able to physically change position within the genome. Two speakers addressed the role of these 'jumping genes' in early development. Juan Vaquerizas (Max Planck Molecular Biomedicine, Germany) used a low input version of the $\mathrm{Hi}-\mathrm{C}$ method (Díaz et al., 2018) - a high-throughput tool that captures the DNA conformation in the nucleus - to analyze chromatin interactions. He and his colleagues found that the murine endogenous retroviral element (MuERV-L/MERVL) family of transposable elements drives the 3D reorganization of the genome in the early mouse embryo (Kruse et al., 2019 preprint). Jacqueline Mermoud (University of Marburg, Germany) discussed the importance of the regulation of endogenous retroviruses in ESCs and showed that their silencing is tightly controlled through ATPdependent chromatin remodeling (Sachs et al., 2019). That protein expression can be controlled at the level of translation rather than transcription was the focus of Olena Zhulyn, a postdoctoral fellow in the Barna laboratory (Stanford University, USA). She showed that translation efficiency plays an important role during both mouse development (Fujii et al., 2017) and regeneration in the axolotl.

Although mouse embryos are key for understanding early developmental processes, they differ quite substantially from primate and human embryos. For example, while the early mouse embryo forms an egg cylinder, early primate and human embryos adopt a flat disc shape. Thorsten Boroviak (University of Cambridge, UK) is therefore aiming to generate an in vivo reference atlas of development in the marmoset, which shares early developmental features with humans. He presented data on laser-captured samples from marmoset uterus sections with embryos that were $3 \mathrm{D}$ reconstructed and analyzed by Shotsequencing, building upon previous work using single cell RNAsequencing (scRNA-seq) to extract a primate-specific signature (Boroviak et al., 2018). His ultimate goal to use these data to generate an in vitro model will still require some time, but will provide important information that cannot be obtained using mouse embryos.

\section{Keynote lecture: Janet Rossant}

In her keynote lecture, Janet Rossant (The Hospital for Sick Children, University of Toronto, Canada) addressed how cells transition from totipotency to pluripotency in the early embryo. Pointing out that the preimplantation embryo represents a 'beautiful self-organizing organoid' to study cell fate transitions, she showed data on the earliest lineage segregation event - the separation of trophectoderm (TE) from the inner cell mass (ICM). This process has been shown to involve position-dependent Hippo signaling. To address the timing of this lineage commitment and gene expression changes, Rossant presented published work that used single cell sequencing and the expression of Cdx2-eGFP as a readout of Hippo signaling (Posfai et al., 2017). Specifically, she reported that the TE and ICM are specified by the 32-cell stage, also reflected by gene expression profiles (i.e. via RNA-seq). This study further showed that the TE is committed to its fate ahead of the ICM. In order to improve mouse genetics and gene editing, Rossant further described a newly developed homologous recombination method that can be applied at the two-cell stage [(2C-HR)-CRISPR], which uses a modified biotin streptavidin approach to improve knock-in efficiency to up to $95 \%$ ( $\mathrm{Gu}$ et al., 2018). This technique was used to generate several knock-in reporter lines (e.g. CDX2-eGFP, GATA-mCherry) to perform live imaging and directly read out the dynamics of these early lineage decisions. Rossant concluded her talk with a note of caution, highlighting that examining cell positioning is not sufficient to prove contribution to the TE, but that both position and trophoblast marker expression need to be carefully examined using single-plane optical confocal microscopy.

\section{Programming and reprogramming cell fate}

Reprogramming forces a cell to give up its cellular identity, yet the low efficiency of this process suggests that cells have a cellular memory of gene expression that allows them to retain their original state. Eva Hörmanseder (a postdoctoral fellow in John Gurdon's laboratory, Gurdon Institute, UK) is examining epigenetic roadblocks that might explain this inefficiency in reprogramming. Splitting genes into two categories, those that need to be switched on and those that need to be switched off during reprogramming, she and her colleagues found a specific epigenetic mark (H3K4me3) that needs to be removed in genes that have to be switched off (Hörmanseder et al., 2017). They are now trying to find further epigenetic marks that can predict resistance to reprogramming. Understand the changes that occur during direct reprogramming was also the topic of Ulrich Elling's (IMBA, Austria) presentation. ESCs and iPSCs can form induced neurons (iNs) via overexpression of the TFs Ngn2 or Ascl1, the latter of which also induces mouse embryonic fibroblasts to iNs. Using an inducible tetO system to overexpress these factors in combination with a CRISPR/Cas9 library to knockout around 6000 different genes, Elling's group is screening for genes that lead to the depletion or enrichment of iNs. Sophie Pèron, a postdoc in the Berninger lab (University of Mainz, 
Germany), reported on their successful in vivo reprogramming experiments in postnatal (P5) mice, targeting primarily astrocytes and oligodendrocyte precursor cells using Ascl1, phospho-mutant forms of Ascl1 or Ascl1 together with Bcl2. The last combination yielded interneuron-like cells that could fire at high frequencies. Intriguingly, she reported that these neurons also receive synaptic input from endogenous neurons.

Whether pluripotent stem cells in culture reflect the preimplantation (naïve) state or the post-implantation (primed) state of the early embryo has been a matter of discussion over the years. These two states are different in many aspects, such as their clonogenic potential, methylation status and X-chromosome inactivation status. Marco Pellegrini, a postdoc in the Martello laboratory (University of Padua, Italy), presented their recently published method using microfluidics and mRNA for reprogramming (Giulitti et al., 2019). He highlighted how this method allowed them to generate human iPSCs that have a naïvelike state (i.e. naïve iPSCs; niPSCs). They are currently trying to understand why the microfluidic system is so much more efficient in reprogramming and whether there are factors that are more concentrated in the confined chambers of the microfluidic chip that exert these effects.

In a 'tour de force' approach, the Lander and the Jaenisch laboratories (Broad Institute of MIT and Harvard, and Whitehead Institute, USA) are aiming to create a 'Google map' of reprogramming. Jian Shu, a postdoctoral fellow in these labs, presented their work on scRNA-seq of an incredible 315,000 cells collected during classical reprogramming of mouse embryonic fibroblasts into iPSCs using the Yamanaka factors. They compared serum versus $2 \mathrm{i}$ culture conditions and sampled every $12 \mathrm{~h}$ for 39 time-points during the reprogramming process. As classical ways of analyzing these large datasets, such as pseudotime trajectories, did not work, they had to develop new ways to analyze these data, which they termed Waddington-OT (optimal transport; Schiebinger et al., 2019). Shu further presented unpublished work aiming to generate induced totipotent cells. Notably, he reported that single cell transplantation of these induced totipotent cells into the eight-cell stage of a developing embryo revealed that they can contribute to extra-embryonic tissues such as the yolk sac, i.e. that they do indeed appear to be totipotent.

\section{Organoids and their potential to advance developmental biology and stem cell research}

The stem cell field has changed dramatically with the development of organoid cultures from various cell types. Organoids, and other 'oid' type structures not only provide great in vitro models with which to manipulate and study self-organization, cell-cell interactions and developmental processes, but also have advanced our understanding of human-specific features. Nicolas Rivron (MERLN Institute and Hubrecht Institute, The Netherlands) highlighted the power of using stem cells and organoids to understand early development. By mixing the right number and proportion of mouse ESCs and trophoblast stem cells (TSCs) together in culture, he and his colleagues were able to create synthetic blastocysts: so-called blastoids due to their similarity to naturally derived blastocysts. Blastoids contain the three cell types present in blastocysts and have the potential to implant into the uterus. Interestingly, when mixed together, the ESC and TSC populations crosstalk to self-organize. This was illustrated by scRNA-seq data showing that the blastoid expression pattern is not the sum of the ESC and TSC expression patterns, but involves the creation of new signatures (Rivron et al., 2018), a phenomenon that is still not understood. Rivron is now using these signatures to study blastocyst axis formation and the formation of polar versus mural trophoblasts, aiming to capture in vitro a constrained, 'polar-like' state of TSCs, as an analog to the 'ground state' of ESCs (FriasAldeguer et al., 2019 preprint).

Brain organoids are more frequently being used in a 'Lego-like' fashion to model complex cell-cell interactions between different brain regions. Jimena Andersen, a postdoc in the Pasca laboratory (Stanford University, USA), presented an initial demonstration of organoid assembly in which human iPSCs are separately patterned to generate subdomains of the forebrain and then put together to form assembloids (Birey et al., 2017, Sloan et al., 2018). She showed how these assembloids can be used to model human cortical interneuron migration deficits in vitro, and to uncover migration deficits in cultures derived from patients with a monogenic disorder called Timothy Syndrome. Andersen then presented an unpublished human assembloid platform that involved the generation of cortical, spinal and muscle organoids to generate a functional threecomponent circuit. She showed that cortical cells connect to neurons in spinal organoids, which then connect to human muscle. Optogenetic activation of the cortical organoids led to an increase in muscle contraction, showing connectivity between the different compartments. Further exploring neuronal projections in organoids, Madeline Lancaster (MRC Laboratory of Molecular Biology Cambridge, UK) introduced a new technique for culturing human cerebral organoids at the air-liquid interface, which greatly improves survival and morphology of neurons but leads to the disappearance of stem cells in the ventricular zone. This new technique resulted in the formation of many dense long-range nerve bundles, similar to tracts in the brain, and scRNA-seq revealed distinct projection neuron types whose molecular identity matched their spatial projection. When put in contact with mouse spinal cord tissue that was still connected to muscle, Lancaster and her team observed nerve bundles growing out of the cerebral organoid and making functional connections to the spinal cord, resulting in increased muscle contraction upon stimulation of the cerebral organoid (Giandomenico et al., 2019). Finally, Rana Fetit (a PhD student in David Price and Thomas Pratt's laboratory, University of Edinburgh, UK) showed how already existing scRNA-seq data from fetal tissue and organoids can be used to better understand the effect of chromosomal deletion in autism spectrum disorders, and how CRISPR/Cas9 modified iPSCs grown into organoids can be used to study these deletion effects.

One drawback of growing organoids in culture is their absence of blood vessels, and hence their lack of oxygen and nutrients, which limits organoid size and often causes cell death in the densely packed center. Thus, many researchers have transplanted organoids back into animal models to enable perfusion and to study organoids in an in vivo context. A first step towards solving this issue was shown by Reiner Wimmer, a postdoctoral fellow in the lab of IMBA founding director Joseph Penninger (IMBA, Austria). He presented recently published work on human blood vessel organoids (Wimmer et al., 2019). Starting from human iPSCs, Wimmer and colleagues have developed a protocol that produces functional blood vessel organoids that can transport blood when transplanted into the kidney of mice. They have used these organoids to model human diabetic vasculopathy, which involves thickening of the basement membrane of vessels and is a major cause of vessel dysfunction in diabetes. Not only could they observe such thickening when they cultured the organoids in a high glucose medium containing pro-inflammatory cytokines, but thickening also occurred when they transplanted the 
organoids into diabetic mice and was specific to the humanderived blood vessels, whereas mouse vessels did not show such thickening. They are currently using this new organoid model to screen for small molecules that could prevent basement membrane thickening and might provide new treatments for individuals with diabetes.

\section{Adult stem cells: insights into regeneration and aging}

Two sessions were dedicated to adult stem cells and regeneration. Various examples of adult stem cell systems, ranging from lung stem cells, HSCs and NSCs, to stem cells in the stomach, were covered. Intriguingly, the presented studies often revealed common regulatory principles across stem cell types and niches, such as metabolism or crosstalk with the immune system. Three talks addressed questions on how HSCs respond to challenges through infection and inflammation, as well as aging. Marieke Essers (German Cancer Research Center, Germany) reported how quiescent HSCs react to inflammatory stress. She and her colleagues found that, upon inflammation, HSCs primarily generate megakaryocytes, which produce platelets (Haas et al., 2015). Using an index-scRNA-seq approach, they further discovered that HSCs in the healthy human bone marrow are primed with regard to interferon (IFN) reactivity and show a large heterogeneity, a mechanism that might provide a larger spectrum of cellular responses to various infections. Such a heterogeneous HSC pool, as opposed to a clear lineage hierarchy, is in line with a previous study to which Essers' group contributed (Velten et al., 2017). Focusing on the effect that aging exerts, Elisa Gomez Perdiguero (Institute Pasteur, France) showed that tissue-resident macrophages are specifically lost during aging and sustained inflammation, and cannot be replaced by HSC-derived cells. Although it is not yet clear why there is such a loss, inflammatory and metabolic pathway changes were shown to accompany this phenomenon during aging. Aging also directly influences HSCs by altering their division pattern: Carolina Florian [Center for Regenerative Medicine in Barcelona, Bellvitge Institute for Biomedical Research (IDIBELL), Spain] reported that aged HSCs perform increased symmetric divisions, accompanied by symmetric distribution of the histone mark H4K16ac, which becomes less enriched specifically on chromosome 11 with aging (Grigoryan et al., 2018). Florian further investigated the functional outcome of aged and young HSC divisions with single daughter cell transplantations. Single cell ATAC-seq revealed enrichment for glycolysis and lipid metabolic pathways in young and old daughter stem cells, whereas only young cells were enriched for IFN/ inflammatory signaling (Florian et al., 2018).

Further adding insight into how metabolism influences adult stem cell behavior, Marlen Knobloch (University of Lausanne, Switzerland) suggested that stem cells across tissues might be regulated by very similar metabolic pathways. She showed that altering fatty acid oxidation through a single metabolite, malonylCoA, overrules quiescence signals in NSCs and triggers proliferation (Knobloch et al., 2017). Using this metabolite on HSCs increases their repopulation capacity and also seems to influence cell fate decisions. Interestingly, metabolism and inflammation also appear to be involved in cell fate changes in lung stem cells. Joo-Hyeon Lee (Cambridge Stem Cell Institute, UK) presented work on the dynamics of lung AT2 cells, using in vivo models as well as alveolar organoids (Lee et al., 2017). Upon injury induced with bleomycin, AT2 cells are activated, modulate metabolic pathways and then replace lung epithelial cells (AT1 cells). Intriguingly, experiments on alveolar organoids revealed that IL-1 $\beta$ signaling from macrophages recapitulates the AT2 cell dynamics observed during repair in vivo.

Understanding cell fate was also the focus of two other talks. Using multicolor fate mapping together with scRNA-seq,

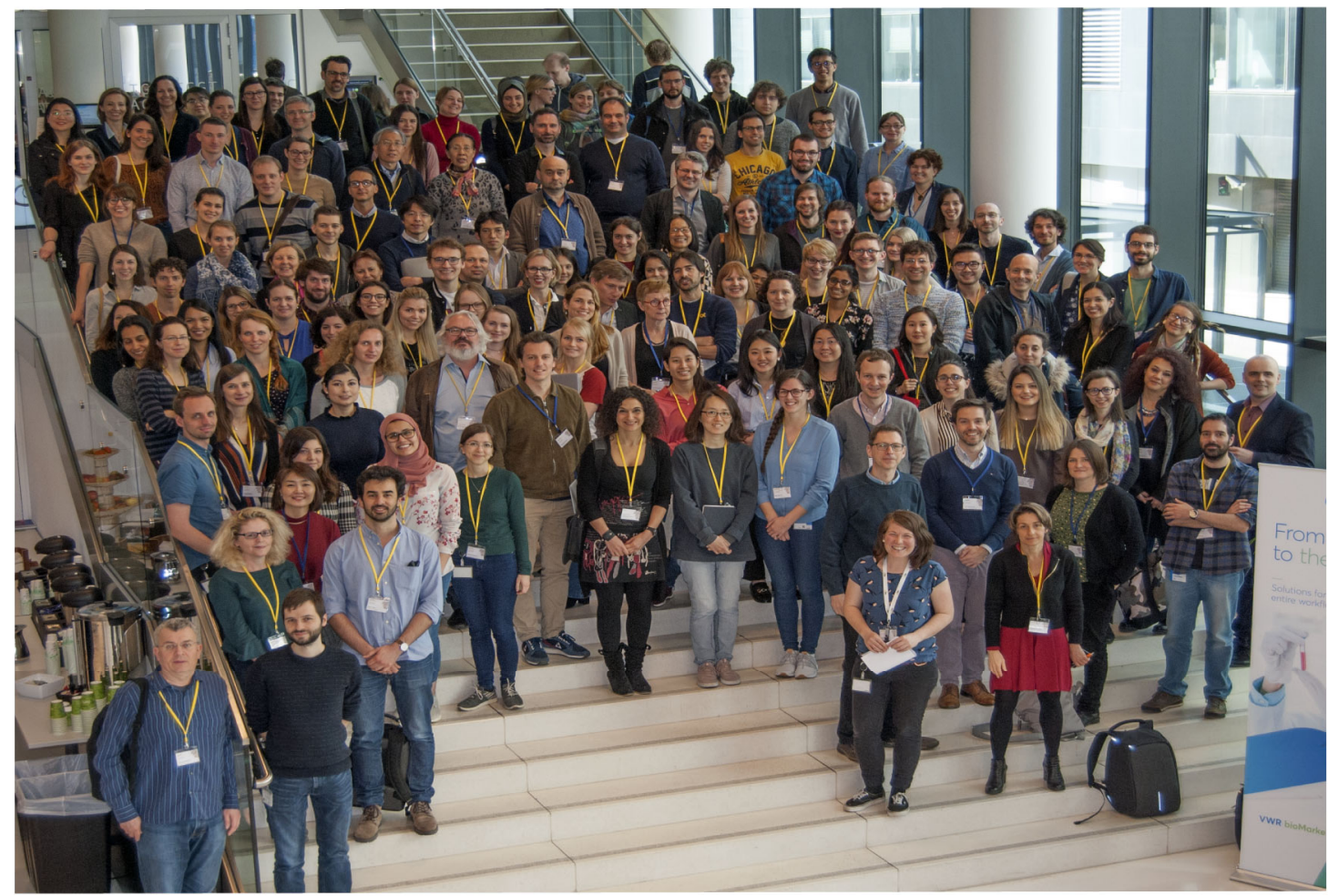

Fig. 2 . The 2019 SY-Stem Symposium. SY-Stem 2019 organizers, speakers and attendees at the BioCenter Vienna in Austria. 
Bon-Kyoung Koo (IMBA, Austria) addressed the origin of stem cells in the stomach, an understudied stem cell compartment. Two compartments, the isthmus and the base, contain stomach stem cells and Bon-Kyoung Koo proposed a novel neutral drift model with barriers to explain the growth of stomach crypts from these two separate compartments. He suggested that such a barrier, which inhibits the rapid expansion of clones, consists of intercalated parietal cells. Moving to stem cell fates in the brain, Gregor Pilz, a postdoc in the lab of Sebastian Jessberger, (University of Zurich, Switzerland), presented an approach to image adult neurogenesis in vivo, enabling observation of NSC fate decisions and lineage progression within the living mouse over the course of weeks (Pilz et al., 2018). In the quest to identify a long-term, self-renewing population within the NSC pool of the dentate gyrus, he used his in vivo imaging paradigm on different $\mathrm{Cre}$-driver lines and obtained a first indication of potential diversity with regard to self-renewing divisions of these stem cells.

In addition to the stem cell types that regenerate tissue within adult mammals, Christian Petersen (University of Chicago, USA), gave examples of the tremendous regenerative capacity in planarians. No matter how many pieces these creatures are cut into, they are able to rebuild a full body out of each piece in a short time, and rescale according to their new size. Petersen explained how a regional 'coordinate system' located in the muscles of planarians expresses regionalized factors that instruct stem cell activity (Hill and Petersen, 2018). He illustrated these operating principles with the example of double-eye mutants, which allowed him to address the homeostasis and regeneration processes separately. With an RNAi screen, they are currently addressing the mechanisms underlying axis scaling and have found mutants that can no longer rescale.

\section{Keynote lecture: Jonas Frisen}

Jonas Frisen (Karolinska Institute, Sweden) showed new data on human adult neurogenesis. Owing to conflicting results from various groups, there has been recent turmoil about whether or not new neurons arise in the human adult brain (for a review, see Kempermann et al., 2018). These contradictory data most likely reflect a general problem when studying human adult stem cells in vivo: the quality of post-mortem tissue is of utmost importance and is a large source of variability. Frisen's group pioneered a way of overcoming these problems by using a method called carbon dating, typically used in archeology to determine the age of historic specimens. Owing to above-ground nuclear bomb testing in the early 1960 s, the amount of ${ }^{14} \mathrm{C}$ in the atmosphere increased sharply and saw a steep fall again after the above-ground test ban a few years later. This unnaturally high ${ }^{14} \mathrm{C}$ content in the atmosphere was gradually incorporated into biomass and allows 'birthdating' of cells that were generated at various times after the start of the tests. Frisen and colleagues already have published many papers using this birthdating method, and have provided evidence that adult neurogenesis does indeed occur throughout life in humans (Bergmann et al., 2012, 2015; Spalding et al., 2013). They have now added more samples to these analyses, reaching more than 100 individuals. Frisen also presented new data (Yeung et al., 2019) on how carbon dating can be useful to understand the processes occurring in individuals with multiple sclerosis. Contrary to what is observed in animal models, where new oligodendrocytes can be formed after demyelination and contribute to remyelination, the turnover rate of human oligodendrocytes seems to be minimal after childhood. These findings have important implications for treatment approaches, suggesting that, given this minimal turnover, a large emphasis should be placed on salvaging mature oligodendrocytes in such patients.

\section{Concluding remarks}

Intense but exciting, the 3-day SY-Stem meeting was hugely successful in bringing together young stem cell researchers (Fig. 2), enabling networking and promoting the exchange of ideas in a friendly environment. We congratulate the organizers for this highquality meeting and hope that this tradition will continue next year with the 3rd annual meeting of SY-Stem.

\section{Acknowledgements}

We thank Iza Kaminski for the permission to use the SY-Stem meeting poster in this publication and the IMBA for the photograph of meeting attendees. We further thank all the speakers for letting us discuss their unpublished work in this Meeting Review.

\section{Competing interests}

The authors declare no competing or financial interests.

\section{Funding}

G.A.P.'s research is supported by funding from a European Molecular Biology Organization long-term fellowship; M.K.'s research is supported by funding from the University of Lausanne, Switzerland and by the Swiss National Science Foundation.

\section{References}

Bergmann, O., Liebl, J., Bernard, S., Alkass, K., Yeung, M. S. Y., Steier, P. Kutschera, W., Johnson, L., Landén, M., Druid, H. et al. (2012). The age of olfactory bulb neurons in humans. Neuron 74, 634-639. doi:10.1016/j.neuron. 2012.03.030

Bergmann, O., Spalding, K. L. and Frisén, J. (2015). Adult neurogenesis in humans. Cold Spring Harb. Perspect Biol. 7, a018994. doi:10.1101/cshperspect.a018994

Birey, F., Andersen, J., Makinson, C. D., Islam, S., Wei, W., Huber, N., Fan, H. C. Metzler, K. R. C., Panagiotakos, G., Thom, N. et al. (2017). Assembly of functionally integrated human forebrain spheroids. Nature 545, 54-59. doi:10. 1038/nature22330

Boroviak, T., Stirparo, G. G., Dietmann, S., Hernando-Herraez, I., Mohammed, H., Reik, W., Smith, A., Sasaki, E., Nichols, J. and Bertone, P. (2018). Single cell transcriptome analysis of human, marmoset and mouse embryos reveals common and divergent features of preimplantation development. Development 145, dev167833. doi:10.1242/dev.167833

Bourque, G., Burns, K. H., Gehring, M., Gorbunova, V., Seluanov, A., Hammell, M., Imbeault, M., Izsvák, Z., Levin, H. L., Macfarlan, T. S. et al. (2018). Ten things you should know about transposable elements. Genome Biol. 19:199, 1-112. doi:10.1186/s13059-018-1577-z

Díaz, N., Kruse, K., Erdmann, T., Staiger, A. M., Ott, G., Lenz, G. and Vaquerizas, J. M. (2018). Chromatin conformation analysis of primarypatient tissue using a low input Hi-C method. Nat. Commun. 9, 4938. doi:10.1038/s41467-018-06961-0

Festuccia, N., Owens, N., Papadopoulou, T., Gonzalez, I., Tachtsidi, A., Vandoermel-Pournin, S., Gallego, E., Gutierrez, N., Dubois, A., CohenTannoudji, M. et al. (2019). Transcription factor activity and nucleosome organization in mitosis. Genome Res. 29, 250-260. doi:10.1101/gr.243048.118

Florian, M. C., Klose, M., Sacma, M., Jablanovic, J., Knudson, L., Nattamai, K. J., Marka, G., Vollmer, A., Soller, K., Sakk, V. et al. (2018). Aging alters the epigenetic asymmetry of HSC division. PLoS Biol. 16, e2003389. doi:10.1371/ journal.pbio.2003389

Frias-Aldeguer, J., Kip, M., Vivié, J., Li, L., Alemany, A., Korving, J., Darmis, F. van Oudenaarden, A., van Blitterswijk, C. A., Geijsen, N. et al. (2019). Embryonic signals perpetuate polar-like trophoblast stem cells and pattern the blastocyst axis. bioRxiv 510362

Fujii, K., Shi, Z., Zhulyn, O., Denans, N. and Barna, M. (2017). Pervasive translational regulation of the cell signalling circuitry underlies mammalian development. Nat. Commun. 8, 1-13. doi:10.1038/s41467-016-0009-6

Giandomenico, S. L., Mierau, S. B., Gibbons, G. M., Wenger, L. M. D., Masullo, L., Sit, T., Sutcliffe, M., Boulanger, J., Tripodi, M., Derivery, E. et al. (2019) Cerebral organoids at the air-liquid interface generate diverse nerve tracts with functional output. Nat. Neurosci. 22, 1-17. doi:10.1038/s41593-019-0350-2

Giulitti, S., Pellegrini, M., Zorzan, I., Martini, P., Gagliano, O., Mutarelli, M., Ziller, M. J., Cacchiarelli, D., Romualdi, C., Elvassore, N. et al. (2019). Direct generation of human naive induced pluripotent stem cells from somatic cells in microfluidics. Nat. Cell Biol. 21, 1-19. doi:10.1038/s41556-018-0254-5

Grigoryan, A., Guidi, N., Senger, K., Liehr, T., Soller, K., Marka, G., Vollmer, A. Markaki, Y., Leonhardt, H., Buske, C. et al. (2018). LaminA/C regulates epigenetic and chromatin architecture changes upon aging of hematopoietic stem cells. Genome Biol. 19:189, 1-121. doi:10.1186/s13059-018-1557-3 
Gu, B., Posfai, E. and Rossant, J. (2018). Efficient generation of targeted large insertions by microinjection into two-cell-stage mouse embryos. Nat. Biotechnol. 36, 632-637. doi:10.1038/nbt.4166

Haas, S., Hansson, J., Klimmeck, D., Loeffler, D., Velten, L., Uckelmann, H., Wurzer, S., Prendergast, Á. M., Schnell, A., Hexel, K. et al. (2015) Inflammation-induced emergency megakaryopoiesis driven by hematopoietic stem cell-like megakaryocyte progenitors. Cell Stem Cell 17, 422-434. doi:10. 1016/j.stem.2015.07.007

Hayashi, K., Hikabe, O., Obata, Y. and Hirao, Y. (2017). Reconstitution of mouse oogenesis in a dish from pluripotent stem cells. Nat. Protoc. 12, 1733-1744 doi:10.1038/nprot.2017.070

Hikabe, O., Hamazaki, N., Nagamatsu, G., Obata, Y., Hirao, Y., Hamada, N., Shimamoto, S., Imamura, T., Nakashima, K., Saitou, M. et al. (2016). Reconstitution in vitro of the entire cycle of the mouse female germ line. Nature 539, 299-303. doi:10.1038/nature20104

Hill, E. M. and Petersen, C. P. (2018). Positional information specifies the site of organ regeneration and not tissue maintenance in planarians. Elife 7, e33680. doi: 10.7554/eLife. 33680

Hörmanseder, E., Simeone, A., Allen, G. E., Bradshaw, C. R., Figlmüller, M., Gurdon, J. and Jullien, J. (2017). H3K4 Methylation-dependent memory of somatic cell identity inhibits reprogramming and development of nuclear transfer embryos. Cell Stem Cell 21, 135-143.e6. doi:10.1016/j.stem.2017.03.003

Kempermann, G., Gage, F. H., Aigner, L., Song, H., Curtis, M. A., Thuret, S., Kuhn, H. G., Jessberger, S., Frankland, P. W., Cameron, H. A. et al. (2018) Human adult neurogenesis: evidence and remaining questions. Cell Stem Cell 23 25-30. doi:10.1016/j.stem.2018.04.004

Knobloch, M., Pilz, G.-A., Ghesquière, B., Kovacs, W. J., Wegleiter, T., Moore, D. L., Hruzova, M., Zamboni, N., Carmeliet, P. and Jessberger, S. (2017). A Fatty acid oxidation-dependent metabolic shift regulates adult neural stem cell activity. Cell Rep. 20, 2144-2155. doi:10.1016/j.celrep.2017.08.029

Kruse, K., Díaz, N., Enriquez-Gasca, R., Gaume, X., Torres-Padilla, M.-E. and Vaquerizas, J. M. (2019). Transposable elements drive reorganisation of 3D chromatin during early embryogenesis. bioRxiv 523712.

Lee, J.-H., Tammela, T., Hofree, M., Choi, J., Marjanovic, N. D., Han, S., Canner, D., Wu, K., Paschini, M., Bhang, D. H. et al. (2017). Anatomically and functionally distinct lung mesenchymal populations marked by Lgr5 and Lgr6. Cell 170, 1149-1156. doi:10.1016/j.cell.2017.07.028
Pilz, G.-A., Bottes, S., Betizeau, M., Jörg, D. J., Carta, S., Simons, B. D., Helmchen, F. and Jessberger, S. (2018). Live imaging of neurogenesis in the adult mouse hippocampus. Science 359,658-662. doi:10.1126/science.aao5056

Posfai, E., Petropoulos, S., de Barros, F. R. O., Schell, J. P., Jurisica, I., Sandberg, R., Lanner, F. and Rossant, J. (2017). Position- and Hippo signalingdependent plasticity during lineage segregation in the early mouse embryo. Elife 6, e22906. doi:10.7554/eLife.22906

Rivron, N. C., Frias-Aldeguer, J., Vrij, E. J., Boisset, J.-C., Korving, J., Vivié, J. Truckenmüller, R. K., van Oudenaarden, A., van Blitterswijk, C. A. and Geijsen, N. (2018). Blastocyst-like structures generated solely from stem cells. Nature 557, 106-111. doi:10.1038/s41586-018-0051-0

Sachs, P., Ding, D., Bergmaier, P., Lamp, B., Schlagheck, C., Finkernagel, F., Nist, A., Stiewe, T. and Mermoud, J. E. (2019). SMARCAD1 ATPase activity is required to silence endogenous retroviruses in embryonic stem cells. Nat Commun. 10, 1-16. doi:10.1038/s41467-019-09078-0

Schiebinger, G., Shu, J., Tabaka, M., Cleary, B., Subramanian, V., Solomon, A., Gould, J., Liu, S., Lin, S., Berube, P. et al. (2019). Optimal-transport analysis of single-cell gene expression identifies developmental trajectories in reprogramming. Cell 176, 928-943.e22. doi:10.1016/j.cell.2019.01.006

Sloan, S. A., Andersen, J., Paşca, A. M., Birey, F. and Paşca, S. P. (2018) Generation and assembly of human brain region -specific three-dimensional cultures. Nat. Protoc. 13, 2062-2085. doi:10.3791/1499

Spalding, K. L., Bergmann, O., Alkass, K., Bernard, S., Salehpour, M., Huttner, H. B., Boström, E., Westerlund, I., Vial, C., Buchholz, B. A. et al. (2013) Dynamics of hippocampal neurogenesis in adult humans. Cell 153, 1219-1227. doi:10.1016/j.cell.2013.05.002

Velten, L., Haas, S. F., Raffel, S., Blaszkiewicz, S., Islam, S., Hennig, B. P., Hirche, C., Lutz, C., Buss, E. C., Nowak, D. et al. (2017). Human haematopoietic stem cell lineage commitment is a continuous process. Nat. Cell Biol. 19, 271-281. doi:10.1038/ncb3493

Wimmer, R. A., Leopoldi, A., Aichinger, M., Wick, N., Hantusch, B., Novatchkova, M., Taubenschmid, J., Hämmerle, M., Esk, C., Bagley, J. A. et al. (2019). Human blood vessel organoids as a model of diabetic vasculopathy. Nature 565, 1-34. doi:10.1038/s41586-018-0858-8

Yeung, M. S. Y., Djelloul, M., Steiner, E., Bernard, S., Salehpour, M., Possnert, G., Brundin, L. and Frisén, J. (2019). Dynamics of oligodendrocyte generation in multiple sclerosis. Nature 566, 1-20. doi:10.1038/s41586-018-0842-3 